\title{
As classificações de atividades econômicas e de produtos aplicadas à indústria química
}

\section{Suzana Borschiver}

\section{Escola de Química da UFRJ}

O presente artigo tem como objetivo apresentar conceitos acerca das classificações industriais pertencentes à "Família Internacional de Classificações Econômicas e Sociais", que abrangem atividades econômicas, produtos, educação, etc.; e demonstrar, particularmente, como as classificações econômicas de atividades e de produtos são aplicadas à cadeia produtiva da indústria química. Para isto, foram selecionadas as principais classificações atividades e produtos pertencentes aos Sistemas de Informação Estatística dos países europeus e das Américas.

Palavras-chave: Classificação industrial; Sistema de Informação Estatística; Análise setorial; Informação estatística; Indústria química; Produtos químicos; Cadeia produtiva.

\section{Classifications of economical activities and products applied to the chemistry industry}

The present article aims at presenting concepts about industrial classifications of the "International Family of Economic and Social Classifications", which comprises economical activities, products, education, etc.; it also intends to demonstrate how the economical classifications of activities and products are applied, particularly, to the productive chain of the chemistry industry. For this, the main classifications of activities and products from the Systems of Statistical Information of European and American countries were selected. 
Keywords: Industrial classification; Systems of Statistical Information; Sectorial analysis; Statistical information; Chemical industry; Chemical products; Productive chain.

\section{Introdução}

O "Sistema de classificações de atividades econômicas e produtos" foi a linguagem comum adotada pelos atores envolvidos nas práticas mercantilistas (países, empresas, indivíduos etc.), para permitir a adequada identificação dos produtos e das atividades integrantes das relações comerciais nacionais e internacionais dos países nelas envolvidos, além de fornecer instrumentos para o planejamento de políticas de desenvolvimento econômico (BORSCHIVER; WONGTSCHOWSKI; ANTUNES, 2004).

As classificações de atividades econômicas conectam-se ao conjunto de agentes do processo de produção, estando distribuídas em diferentes modalidades: produtivas (como bens e serviços de manutenção/reparação de máquinas), e as atividades direta e indiretamente associadas ao processo produtivo (BORSCHIVER, 2002). As classificações de produtos (incluindo os químicos), integrantes do conjunto dos bens e serviços, promovem o agrupamento dos dados segundo as características físicas, tipos de insumo, etapas do processo produtivo, origem no processo produtivo e mercado ou finalidade de uso.

\section{A família internacional de classificações econômicas}

Os países utilizam como padrão de referência para as classificações de atividades econômicas e produtos a denominada "Família Internacional de Classificações Econômicas e Sociais", sob regulação dos organismos pertencentes à Organização das Nações Unidas (ONU); e no contexto nacional essas classificações integram o "Sistema de Informação Estatística" (SIE). A Família Internacional engloba três principais níveis hierárquicos de classificações (BORSCHIVER; WONGTSCHOWSKI; ANTUNES, 2004):

a)Classificações de referência - resultantes dos acordos internacionais aprovados pela United Nation Statistical Commission ou outro órgão intergovernamental competente, dependendo do tema envolvido. As classificações de referência são de ampla aceitação e concordância oficial, sendo aprovadas e recomendadas como referências (guidelines) para o desenvolvimento de outras classificações, tanto em relação à estrutura quanto em relação ao caráter e definição das categorias;

b)Classificações derivadas - baseadas nas classificações de referência e preparadas tanto pela (a) adoção das categorias e da estrutura das classificações de referência, às vezes incluindo um 
detalhamento adicional àquele fornecido por ela, quanto (b) por meio da reorganização ou agregação de itens de uma ou mais classificações de referência; e

c)Classificações relacionadas - referem-se parcialmente às classificações de referência, ou são associadas a estas apenas em alguns níveis específicos de sua estrutura.

Para a realização deste trabalho, foram selecionadas as classificações de atividade e de produtos (referências, derivadas e relacionadas) usadas nos SIE dos países europeus e das Américas, como será visto nos próximos parágrafos.

\section{Principais classificações internacionais de atividades econômicas e de produtos e sua aplicação à indústria química}

\subsection{Classificações de atividades econômicas}

- International Standard Industrial Classification of All Economic Activities (ISIC):

A ISIC é uma classificação-referência de atividades econômicas gerida pelo United Nation Economic Statistics and Classifications Section (UNSD). A TAB. 1, a seguir, mostra a atual estrutura da ISIC Revision 4, ou ISIC Rev 4.

TABELA 1

Estrutura da Classificação ISIC Rev 4 (International Standard Industrial Classification of All Economic Activities)

\begin{tabular}{lcc}
\hline \multicolumn{1}{c}{ Nível } & Codificação & oo de itens \\
\hline \hline Seções & 1 caractere alfabético (A-U) & 21 \\
Divisões & 2 dígitos & 88 \\
Grupos & 3 dígitos & 238 \\
Classes & 4 dígitos & 420 \\
\hline
\end{tabular}

Fonte: Nações Unidas (2007a).

No QUADRO 1, exemplifica-se o processo de codificação aplicado à Indústria Química, através dos níveis compreendidos na Categoria das Indústrias de Transformação do sistema de classificação ISIC.

QUADRO 1

Exemplificação do processo de codificação do Sistema de Classificação ISIC Rev 4 (International

Standard Industrial Classification of All Economic Activities) aplicado à Indústria Química

C - Indústrias de transformação (SEÇÃO)

20 - Manufaturas de produtos químicos (DIVISÃO)

201 - Produção de químicos básicos, fertilizantes, plásticos e borracha sintética (GRUPO)

2011 - Produção de químicos básicos (CLASSE)

Fonte: Elaborado a partir de Nações Unidas (2007b). 
A manufatura de produtos químicos encontra-se distribuída ao longo da Divisão 20 da Categoria C (Indústrias de Transformação), nas Classes pertencentes aos Grupos 201 a 203. O Grupo ISIC 201 refere-se à manufatura de produtos químicos básicos (Classe 2011), fertilizantes (Classe 2012), e plásticos e borracha sintética em suas formas primárias (Classe 2013). A Classe ISIC 2011 compreende os produtos químicos básicos inorgânicos e orgânicos em geral, como os gases industriais, tinturas e pigmentos, água destilada, produtos petroquímicos derivados do refino do petróleo etc., ressalvando os compostos químicos localizados nas outras classes da ISIC.

O Grupo ISIC 202 trata da fabricação dos produtos químicos não englobados no grupo anterior (ISIC 201), estendendo-se da Classe ISIC 2021 até a 2029. A Classe ISIC $2021 \mathrm{faz}$ referência à produção de pesticidas e outros agroquímicos, além dos produtos antibrotamento, reguladores do crescimento de plantas e desinfetantes agrícolas. A Classe ISIC 2022 trata da produção de tintas e vernizes junto com revestimentos, tintas de impressão e mastiques, mas excluindo as tinturas e pigmentos (posicionados na Classe ISIC 2011) e as tintas para desenho e escrita (Classe ISIC 2029, produção de outros produtos químicos). A Classe ISIC 2023 unifica, em uma mesma classificação, a produção de compostos distintos como sabões, detergentes e preparações de limpeza, cosméticos, perfumes e preparações de toalete. 0 Grupo ISIC 203 descreve a manufatura de fibras artificiais e sintéticas.

A última Classe do Grupo 202, a ISIC 2029, trata da produção de outros produtos químicos, por exemplo propelentes em pó, explosivos e produtos pirotécnicos, colas e adesivos, produtos fotográficos, óleos essenciais, catalisadores, etc.

Além da Divisão 20, a Divisão 21 aborda a produção de produtos químico-farmacêuticos e de uso medicinal, através do seu grupo ISIC 210. Outras Divisões (ISIC 22 a 24) privilegiam produtos acabados elaborados com os compostos químicos descritos nos grupos e classes dos parágrafos anteriores, como plásticos e borrachas.

- Nomenclature Générale des Activités Économiques dans les Communautées Européennes (NACE):

Até o final da década de 1960, os países europeus trabalhavam com nomenclaturas próprias, o que dificultava a produção de dados comparáveis. Em 1970, para resolver esta situação no âmbito da União Européia, foi criada a Nomenclature Générale des Activités Économiques dans les Communautées Européennes (NACE).

Em 1990, para substituir a NACE 70, foi elaborada a NACE Rev 1, com base na ISIC Rev 3, tornando-se uma classificação-derivada desta, e tendo como gestor o Statistical Office of the European Communities. Em 
2002, foi promovida a segunda atualização deste sistema, originando a NACE Rev 2, cuja estrutura hierárquica é mostrada na TAB. 2 a seguir.

TABELA 2

Estrutura da Classificação NACE Rev 2 (Nomenclature Générale des Activités Économiques dans les Communautées Européennes)

\begin{tabular}{lcc}
\hline \multicolumn{1}{c}{ Nível } & Codificação & № de itens \\
\hline \hline Seções & 1 caractere alfabético (A-Q) & 21 \\
Divisões & 2 dígitos & 88 \\
Grupos & 3 dígitos & 272 \\
Classes & 4 dígitos (01.11 a 99.00) & 615 \\
\hline
\end{tabular}

Fonte: União Européia (2007a).

O QUADRO 2, abaixo, exemplifica o processo de codificação aplicado ao Sistema NACE Rev 2.

QUADRO 2

Exemplificação do processo de codificação do Sistema de Classificação NACE Rev 2 (Nomenclature Générale des Activités Économiques dans les Communautées Européennes) aplicado à Indústria Química

C - Indústrias de transformação (SEÇÃ̃)

20 - Manufaturas de produtos químicos (DIVISÃO)

20.1 - Produção de químicos básicos, fertilizantes, plásticos e borracha sintética (GRUPO)

20.11 - Produção de gases industriais (CLASSE)

Fonte: Elaborado a partir de União Européia (2007b).

Sendo uma classificação-derivada da ISIC, e utilizando estrutura similar à mesma, a NACE apresenta uma distribuição de divisões, de grupos e de classes, compatível com a sua classificação-referência. A manufatura de produtos químicos está na Divisão 20 da Categoria C (Indústrias de transformação), nas Classes pertencentes aos Grupos 20.1 a 20.6. As matérias abrangidas pelas Classes NACE 20.15 (produção de produtos fertilizantes), NACE 20.20 (produção de pesticidas e outros agroquímicos), NACE 20.30 (produção de tintas e vernizes), pelo Grupo NACE 20.5 (produção de outros produtos químicos) e Grupo NACE 20.6 (manufatura de fibras artificiais e sintéticas), equivalem aos respectivos grupos e classes da ISIC. Também obedecendo à ISIC, a produção de pesticidas e outros agroquímicos (Classe NACE 20.20) abrange os produtos antibrotamento, reguladores do crescimento de plantas e desinfetantes agrícolas, e a produção de tintas e vernizes engloba os revestimentos, tintas de impressão e mastiques, mas exclui as tinturas e pigmentos (Classe NACE 20.12). Contudo, as tintas para desenho e escrita continuam sendo alocadas na produção de outros produtos químicos (Classe NACE 20.59).

$\mathrm{Na} N A C E$, divergindo da sua classificação-referência, os compostos químicos considerados "inorgânicos básicos" (por exemplo os elementos 
químicos, os ácidos inorgânicos, hidróxidos alcalinos, água destilada, etc.) encontram-se na Classe NACE 20.13, separados dos compostos químicos "orgânicos básicos" (localizados na NACE 20.14), ressalvando os compostos químicos localizados nas outras classes. Gases industriais e tinturas e pigmentos não se encontram listados na NACE 20.14, mas sim em Classes próprias, respectivamente, NACE 20.11 e NACE 20.12.

No Grupo NACE 20.4, sabões, detergentes e preparações de limpeza, cosméticos, perfumes e preparações de toalete encontram-se distribuídos nas Classes: NACE 20.41, para os sabões, detergentes e preparações de limpeza; e NACE 20.42, para cosméticos, perfumes e preparações de toalete. A produção de resinas plásticas e de borracha sintética encontra-se, respectivamente, nas classificações NACE 20.16 e NACE 20.17. A exemplo da ISIC 2029, as Classes NACE 20.51 a 20.59 tratam da produção dos demais produtos químicos.

Além da Divisão 20, a Divisão 21 aborda a produção de produtos químico-farmacêuticos e de uso medicinal, através dos seus Grupos NACE 21.1 (Manufatura de produtos farmacêuticos básicos) e 21.2 (Manufatura de preparações farmacêuticas). Outras Divisões (NACE 22 a 24) privilegiam produtos acabados elaborados com os compostos químicos descritos nos grupos e classes dos parágrafos anteriores. Os produtos petroquímicos derivados do refino do petróleo não se encontram alistados na Divisão 20, mas sim na Divisão 19, Classe 19.20.

- North American Industry Classification System (NAICS):

O North American Industry Classification System (NAICS) foi desenvolvido em conjunto pelo U.S. Economic Classification Policy Committee (ECPC) do Office of Management and Budget (OMB), Statistics Canada e Instituto Nacional de Estadística, Geografia e Informática do México (INEGI), utilizando a ISIC Rev 3 como referência, e tornando-se uma classificação-relacionada à mesma. O NAICS substituiu os respectivos sistemas de classificação dos países citados, entrando em vigor, no Canadá e nos EUA, no ano de 1997, e, no México, no ano de 1998.

Os gestores dos NAICS-México, NAICS-Canadá e NAICS-USA são, respectivamente, a 'Sección Econômica de las Clasificaciones de Instituto Nacional de Estadística, Geografia e Informática do México (INEGI)', 'Standards Division of the Statistics Canada' e 'Service Sector Statistics Division of the U.S. Bureau of the Census', e seus dados estatísticos podem ser comparados entre si até o nível de "Indústria NAICS". A partir do nível "Indústria Nacional NAICS", as classificações variam de acordo com o tipo de organização industrial de cada país.

A atual estrutura hierárquica genérica da NAICS encontra-se na TAB. 3 a seguir. 
TABELA 3

Estrutura da Classificação NAICS (North American Industry Classification System)

\begin{tabular}{lcc}
\hline \multicolumn{1}{c}{ Nível } & Codificação & № de itens \\
\hline \hline Setores & 2 dígitos & --- \\
Subsetores & 3 dígitos & --- \\
Grupos de indústria & 4 dígitos & --- \\
Grupos de indústria internacional & 5 dígitos & 713 \\
Grupos de indústria nacional & 6 dígitos & 1.170 \\
\hline
\end{tabular}

Fonte: Nações Unidas (2007c).

O QUADRO 3, abaixo, exemplifica o processo de codificação do Sistema NAICS-USA.

$$
\text { QUADRO } 3
$$

Exemplificação do processo de codificação do Sistema de Classificação NAICS-USA (North American Industry Classification System of United States of America) aplicado à Indústria Química

31-33 - Indústrias de transformação (SETOR)

325 - Manufaturas de produtos químicos (SUBSETOR)

3251 - Produção de químicos básicos (GRUPO DE INDÚSTRIA)

32513 - Indústria de pigmentos e corantes sintéticos (Grupo de Indústria Internacional)

325131 - Indústria de pigmentos e corantes inorgânicos (Grupo de Indústria Nacional Norte-americana)

Fonte: Elaborado a partir de U.S. CENSUS BUREAU (2007).

Embora seja uma classificação-relacionada à ISIC, a estrutura utilizada na NAICS-USA difere da sua classificação-referência, a começar pelo número de dígitos utilizados, ou seja, seis, enquanto a ISIC utiliza um sistema de quatro dígitos. A manufatura de produtos químicos encontra-se distribuída ao longo do Subsetor 325. Os Grupos de Indústria Internacionais NAICS 32518 e 32519 referem-se, respectivamente, à produção de produtos químicos inorgânicos básicos e orgânicos básicos, ressalvando os compostos químicos localizados nas demais classes. Gases industriais e tinturas e pigmentos não se encontram nos grupos supracitados, mas sim em Grupos de Indústria Internacional próprios, respectivamente, a NAICS 32512 e a NAICS 32513.

A produção de tintas e vernizes (Grupo de indústria NAICS 3255) também abrange solventes, removedores, outros revestimentos tais como lacas, esmaltes, repelentes à água, mas exclui tinturas e pigmentos. Contudo, as tintas para desenho e escrita continuam sendo alocadas na produção de outros produtos químicos (Grupo de indústria internacional NAICS 32599). Diferente da ISIC e NACE, os adesivos são computados em uma categoria própria, e não compilados na "produção de outros produtos químicos", pertencendo ao mesmo Grupo de indústria das tintas (NAICS 32552).

A produção de resinas plásticas, borracha sintética e fibras artificiais e sintéticas encontram-se, no Grupo de indústria NAICS 3252, 
distribuídos, respectivamente, nas classificações NAICS 325211, NAICS 325212 e NAICS 32522. A produção de sabões, detergentes e preparações de limpeza está na NAICS 32561 e a produção de cosméticos, perfumes e preparações de toalete na NAICS 32562.

Embora computados no Grupo de indústria NAICS 3253, pesticidas, fertilizantes e outros agroquímicos são distribuídos em dois grupos de indústria internacionais distintos: NAICS 32531, para Fertilizantes, e NAICS 32532, para Pesticidas e outros agroquímicos. A exemplo da ISIC 2029, o NAICS 3259 trata da produção de outros produtos químicos.

Diferente das duas Classificações anteriores, a produção de produtos químico-farmacêuticos e de uso medicinal pertence ao mesmo rol da manufaturas de produtos químicos (Subsetor 325), através do Grupo de indústria NAICS 3254. Em semelhança, os produtos petroquímicos derivados do refino do petróleo estão listados no Grupo de indústria internacional 32511 pertencente ao Subsetor 325. Outros subsetores (NAICS 326 a 331 ) privilegiam produtos acabados elaborados a partir dos compostos químicos descritos nos grupos e classes dos parágrafos anteriores.

- Classificação Nacional de Atividades Econômicas (CNAE):

A Classificação Nacional de Atividades Econômicas (CNAE) foi criada em 1994 e é gerenciado no Brasil pelo Instituto Brasileiro de Geografia e Estatística (IBGE). A CNAE é o instrumento de padronização utilizado pelos diversos órgãos da Administração Tributária do Brasil, como a Receita Federal. A partir de acordos internacionais assinados pelo Brasil, foi adotada uma estrutura padronizada baseada na ISIC, tornando-se uma classificação-relacionada à mesma. Atualmente, vigora a versão CNAE 2.0, cuja estrutura hierárquica está na TAB. 4 a seguir.

TABELA 4

Estrutura da Classificação CNAE (Classificação Nacional de Atividades Econômicas)

\begin{tabular}{lcc}
\hline \multicolumn{1}{c}{ Nível } & Codificação & No de itens \\
\hline \hline Seções & 1 caractere alfabético (A-Q) & 17 \\
Divisões & 2 dígitos & 59 \\
Grupos & 3 dígitos & 223 \\
Classes & 4 dígitos & 581 \\
& +1 dígito verificador & \\
Subclasses & +2 dígitos após o dígito verificador & 1.183 \\
\end{tabular}

Fonte: Comissão Nacional de Classificação - CONCLA (2007).

A seguir, no QUADRO 4, é exemplificada a estrutura deste sistema de classificação. 
QUADRO 4

Exemplificação do processo de codificação do Sistema de Classificação CNAE (Classificação Nacional de Atividades Econômicas) aplicado à Indústria Química

C - Indústrias de transformação (SEÇÃO)

20 - Fabricação de produtos químicos (DIVISÃO)

201 - Fabricação de produtos químicos inorgânicos (GRUPO)

2011-8 - Fabricação de cloro e álcalis (CLASSE)

2011-8/00 - Fabricação de cloro e álcalis (SUBCLASSE)

Fonte: Elaborado a partir de CONCLA (2007).

Apesar de ser uma classificação-relacionada à ISIC, sua estrutura também difere desta, empregando um sistema numérico de até sete dígitos, enquanto a ISIC utiliza um sistema exclusivo de quatro dígitos. A manufatura de produtos químicos encontra-se distribuída ao longo da Divisão 20 da Categoria C (Indústrias de transformação), nas Classes e Subclasses pertencentes aos Grupos 201 a 209. Os Grupos CNAE 201 e CNAE 202 referem-se, respectivamente, à produção de produtos químicos inorgânicos básicos e orgânicos básicos, ressalvando os compostos químicos localizados nas outras classes pertencentes à CNAE. Quanto aos fertilizantes e intermediários de fertilizantes, apenas os de natureza inorgânica estão registrados nas Classes CNAE 2012-6 e CNAE 2013-4. Os fertilizantes de natureza orgânica encontram-se na Classe 2099-1 do Grupo 209 (fabricação de outros produtos químicos).

O Grupo CNAE 203 engloba, sob uma mesma classificação, a fabricação de resinas plásticas e borracha sintética. O Grupo CNAE 204 trata da manufatura de fibras artificiais e sintéticas. Na CNAE, os pesticidas são renomeados de "defensivos agrícolas", e sua fabricação está registrada na Classe CNAE 2051-7.

Como na ISIC, tanto a produção de sabões, detergentes e preparações de limpeza quanto os cosméticos, perfumes e preparações de toalete estão unificados no Grupo 206: CNAE 2061-4 - fabricação de sabões e detergentes sintéticos, CNAE 2062-2 - fabricação de produtos de limpeza e polimento, e CNAE 2063-1 - fabricação de cosméticos, produtos de perfumaria e de higiene pessoal. Por sua vez, a fabricação de desinfetantes domissanitários está alocada na Classe CNAE 2052-5, do Grupo 205 (fabricação de defensivos agrícolas e desinfetantes domissanitários).

A produção de tintas e vernizes (Grupo CNAE 207) também abrange esmaltes, lacas, tintas de impressão, impermeabilizantes, solventes e produtos afins. Contudo, a fabricação de corantes e pigmentos se encontra distribuída nas Classes 20.19-3 e 20.29-1. Além disso, a fabricação de tintas de impressão e de tintas para escrever e desenhar estão, respectivamente, nas Classes 20.72-0 e 20.99-1.

Os produtos petroquímicos derivados do refino do petróleo estão listados na Classe 2021-5. A exemplo da ISIC 2029, o Grupo CNAE 209 trata da produção de outros produtos químicos. 
Além da Divisão CNAE 20, a Divisão CNAE 21 aborda a produção de produtos químico-farmacêuticos e de uso medicinal, através dos seus grupos CNAE 211 (fabricação de produtos farmoquímicos) e 212 (fabricação de produtos farmacêuticos). Outras divisões (CNAE 22 a 24) privilegiam produtos acabados elaborados a partir dos compostos químicos descritos nos grupos e classes dos parágrafos anteriores.

\subsection{Classificação de produtos}

- Harmonized Commodity Description and Coding System (HS):

Por volta de 1970, o Customs Cooperation Council (CCC), em conjunto com a United Nation Economic Statistics and Classifications Section (UNSD) e outros órgãos estatísticos nacionais e internacionais, iniciou estudos para reduzir os problemas relativos à codificação e designação de mercadorias. Em 1986, o CCC aprovou o Harmonized Commodity Description and Coding System ou Harmonized System (HS), uma nomenclatura comum de referência para atender aos interesses aduaneiros e estatísticos envolvidos no comércio internacional.

O Sistema HS é uma classificação de produtos de referência que tem como órgão internacional responsável o World Customs Organization (WCO), e compreende um total de 7.466 grupos de produtos identificados, constituindo uma organização sistemática e uniforme de produtos. Atualmente, as estatísticas de exportação e importação são classificadas e publicadas de acordo com o HS, com sua versão mais recente entrando em vigor no segundo semestre de 2007. A atual estrutura hierárquica do sistema de classificação HS é apresentada na TAB. 5 a seguir.

TABELA 5

Estrutura da Classificação HS (Harmonized Commodity Description and Coding System)

\begin{tabular}{lcc}
\hline \multicolumn{1}{c}{ Nível } & Codificação & № de itens \\
\hline \hline Seções & Numerais romanos (I a XXI) & 21 \\
Capítulos & 2 dígitos & 96 \\
Posições & 4 dígitos & -- \\
Categorias & 6 dígitos & --- \\
\hline
\end{tabular}

Fonte: União Européia (2007c).

A seguir, no QUADRO 5, é exemplificada a estrutura deste sistema de classificação.

QUADRO 5

Exemplificação do processo de codificação do Sistema de Classificação HS (Harmonized Commodity Description and Coding System) aplicado à Indústria Química

VI - Produtos das indústrias químicas ou das indústrias conexas (SEÇÃO)

29 - Produtos químicos orgânicos (CAPÍTULO)

CAPÍTULO: 29

POSIÇÃO: 2901 (Hidrocarbonetos Acíclicos)

CATEGORIA: 2901.10 (Hidrocarbonetos Acíclicos Saturados)

Fonte: Elaborado A Partir de União Européia (2007d). 
No âmbito do MERCOSUL (Mercado Comum do Sul), é amplamente utilizada uma classificação-relacionada à HS: a Nomenclatura Comum do Mercosul (NCM). A sistemática de classificação dos códigos na Nomenclatura Comum do Mercosul (NCM) obedece rigidamente à estrutura hierárquica da HS, sendo acrescida de dois dígitos. Desta maneira, comparada à HS, a NCM contém um nível a mais (Subcategorias), e a codificação é composta, no total, por 8 dígitos, como apresentado no QUADRO 5.1.

\section{QUADRO 5.1}

Exemplificação do processo de codificação do Sistema de Classificação NCM (Nomenclatura Comum do Mercosul) aplicado à Indústria Química

VI - Produtos das indústrias químicas ou das indústrias conexas (SEÇÃO)

29 - Produtos químicos orgânicos (CAPÍTULO)

CAPÍTULO: 29

POSIÇÃO: 2901 (Hidrocarbonetos Acíclicos)

CATEGORIA: 2901.10 (Hidrocarbonetos Acíclicos Saturados)

SUBCATEGORIA: 2901.10.00 (Hidrocarbonetos Acíclicos Saturados)

Fonte: Elaborado a partir de MERCOSUL (2007).

Retornando ao Harmonized System, os produtos químicos distribuem-se ao longo dos Capítulos HS 28 a 38 da Seção VI (Produtos das indústrias químicas ou das indústrias conexas). Os Capítulos HS 28 e 29 referem-se, respectivamente aos produtos químicos inorgânicos básicos e orgânicos básicos, ressalvando os compostos químicos localizados nas outras classes. Os produtos químico-farmacêuticos e de uso medicinal são abordados no Capítulo 30, e os fertilizantes no Capítulo HS 31. O Capítulo HS 32 trata das tintas e vernizes em conjunto com esmaltes, lacas, pigmentos, opacificantes, solventes e diluidores, removedores, desenho e escrita, e outros corantes.

O Capítulo HS 33 aborda os cosméticos, perfumes e preparações de toalete, e o Capítulo HS 34, os sabões, detergentes e preparações de limpeza. Os pesticidas encontram-se no Capítulo HS 38 (produtos químicos variados), nas Categorias HS 3808.10 (inseticidas), HS 3808.20 (fungicidas), HS 3808.30 (herbicidas, produtos antibrotamento e reguladores do crescimento de plantas) e HS 3808.40 (desinfetantes).

Os produtos petroquímicos derivados do refino do petróleo encontram-se em outra Seção, a HS V (produtos minerais). As resinas plásticas e de borracha sintética encontram-se na Seção HS VII. O Capítulo 55, pertencente à Seção HS XI, refere-se às fibras artificiais e sintéticas. Outras Seções da HS privilegiam produtos acabados elaborados a partir dos compostos químicos descritos nos grupos e classes dos parágrafos anteriores.

- Central Product Classification (CPC): 
A Central Product Classification (CPC) é um sistema de classificação de referência gerido pela United Nation Economic Statistics and Classifications Section (UNSD). Aprovado em 1997, em sua Version 1.0, substituiu a Provisional Central Product Classification (Provisional CPC), de 1989. Atualmente, está em vigor a versão 1.1 , sendo que a partir do segundo semestre de 2007 vigorará a versão 2.0.

A estrutura hierárquica da CPC Version 1.1 é apresentada na TAB. 6, e as seções de 0 a 4 detalham os mesmos níveis de agregação do Harmonized System (HS).

TABELA 6

Estrutura da Classificação CPC Version 1.1 (Central Product Classification)

\begin{tabular}{lcc}
\hline \multicolumn{1}{c}{ Nível } & Codificação & № de itens \\
\hline \hline Seção & 1 dígito (0-9) & 10 \\
Divisão & 2 dígitos & 70 \\
Grupos & 3 dígitos & 305 \\
Classes & 4 dígitos & 1.167 \\
Subclasses & 5 dígitos & 2.096
\end{tabular}

Fonte: Nações Unidas (2007d).

A seguir, no QUADRO 6, é exemplificada a estrutura deste sistema de classificação.

QUADRO 6

Exemplificação do processo de codificação do Sistema de Classificação CPC Version 1.1

(Central Product Classification) aplicado à Indústria Química

3 - Outros produtos transportáveis, exceto produtos metálicos, maquinário e equipamentos (SEÇÃO)

34 - Produção de químicos básicos (DIVISÃO)

341 - Produção de químicos orgânicos básicos (GRUPO)

3412 - ácidos graxos monocarboxílicos industriais (CLASSE)

34120 - ácidos graxos monocarboxílicos industriais (SUBCLASSE)

Fonte: Elaborado a partir de Nações Unidas (2007e).

Os produtos químicos encontram-se distribuídos ao longo das Divisões 34 (Químicos Básicos) e 35 (Outros produtos químicos; fibras artificiais e sintéticas) da Seção 3 (Outros produtos transportáveis, exceto produtos metálicos, maquinário e equipamentos). Os Grupos CPC 341 e 342 referem-se, respectivamente, aos produtos químicos orgânicos básicos e inorgânicos básicos, ressalvando os compostos químicos localizados nas outras classes.

Os produtos petroquímicos derivados do refino do petróleo encontram-se distribuídos ao longo das Classes pertencentes aos Grupos 344 e 345. O Grupo 346 trata dos fertilizantes e pesticidas, respectivamente nas Classes 3461 e 3462. As resinas plásticas e de borracha sintética encontram-se, respectivamente, sob as classificações CPC 347 e CPC 348. 
O Grupo 351 trata das tintas e vernizes em conjunto com esmaltes, lacas, pigmentos, opacificantes, solventes e diluidores, removedores, desenho e escrita, e outros corantes. Outros compostos químicos corantes são encontrados no Grupo 343.

Diferente das Classificações anteriores, a produção de produtos químico-farmacêuticos e de uso medicinal pertence ao mesmo rol da manufatura de produtos químicos (Grupo CPC 352). O grupo CPC 353 aborda os sabões, detergentes e preparações de limpeza, cosméticos, perfumes e preparações de toalete, através das Subclasses CPC 35310 a 35334; sendo que na CPC 35323 são abordados os perfumes e preparações de toalete, e na CPC 35331 as preparações para desodorização ou odorização de ambientes. O Grupo CPC 355 refere-se às fibras artificiais e sintéticas. Os Grupos 344, 345 e 354 tratam dos demais produtos químicos não listados nos campos anteriores.

Outras Divisões (CPC 36 e 37) privilegiam produtos acabados elaborados a partir dos compostos químicos descritos nos grupos e classes dos parágrafos anteriores.

- Standard International Trade Classification (SITC):

A Standard International Trade Classification Rev 4 é um sistema de classificação de comércio exterior derivada da Harmonized System, tendo como gestor a UNSD (United Nation Economic Statistics and Classifications Section). A partir da sua terceira revisão, sua importância tem sido cada vez menor, devido à ampla utilização do HS em todo o mundo. Na TAB. 7, é apresentada a estrutura hierárquica desta classificação.

TABELA 7

Estrutura da Classificação SITC Rev 4 (Standard International Trade Classification)

\begin{tabular}{lcc}
\hline \multicolumn{1}{c}{ Nível } & Codificação & No de itens \\
\hline \hline Seções & 1 dígito (0-9) & 10 \\
Divisões & 2 dígitos & 67 \\
Grupos & 3 dígitos & 262 \\
Classes & 4 dígitos & 1.023 \\
Subclasses & 5 dígitos & 2.970
\end{tabular}

Fonte: União Européia (2007e).

A seguir (QUADRO 7), exemplifica-se a estrutura da SITC Rev 4. QUADRO 7

Exemplificação do processo de codificação do Sistema de Classificação SITC Rev 4

(Standard International Trade Classification) aplicado à Indústria Química

5 - Produtos químicos e relacionados (SEÇÃO)

51 - Químicos Orgânicos (DIVISÃO)

511 - Hidrocarbonetos e seus derivados halogenados, sulfonados, nitrados ou nitrosados (GRUPO)

511.1 - Hidrocarbonetos acíclicos (SUBGRUPO)

511.11 - Etileno (Subclasse)

Fonte: Elaborado a partir de Nações Unidas (2007f). 
A Standard International Trade Classification (SITC), em sua quarta revisão, apresenta uma distribuição de divisão, grupos e classes compatível com a sua classificação-referência. Os produtos químicos encontram-se distribuídos ao longo da Seção 5, nas Divisões 51 a 59. As Divisões SITC 51 e 52 referem-se, respectivamente aos produtos químicos orgânicos básicos e inorgânicos básicos, ressalvando os compostos químicos localizados nas outras classes.

A Divisão SITC 53 trata das tintas e vernizes em conjunto com esmaltes, lacas, pigmentos, opacificantes, solventes e diluidores, removedores, desenho e escrita, e outros corantes. Os produtos químicofarmacêuticos e de uso medicinal são abordados na Divisão SITC 54.

A Divisão 55 aborda conjuntamente os sabões, detergentes e preparações de limpeza, cosméticos, perfumes e preparações de toalete; sendo que no Grupo SITC 553 trata-se dos perfumes, cosméticos e preparações de toalete, e no SITC 554 dos sabões, detergentes e preparações de limpeza. A Divisão SITC 56 trata dos fertilizantes, e as resinas plásticas estão na Divisão SITC 57. O Grupo SITC 591, pertencente à Divisão SITC 59, aborda os pesticidas.

Os Grupos SITC 516, 524 e a Divisão SITC 59 tratam dos demais produtos químicos não listados nos campos anteriores. A borracha sintética pertence à Divisão SITC 23 e os produtos petroquímicos derivados do refino do petróleo encontram-se distribuídos na Divisão SITC 33. OS Grupos SITC 266 e 267 referem-se às fibras artificiais e sintéticas.

Os produtos acabados elaborados a partir dos compostos químicos descritos nos grupos e classes dos parágrafos anteriores se encontram nas Divisões 36 e 37, dentre outras.

- Statistical Classification of Products by Activity in the European Economic Community (CPA):

A CPA é uma classificação-derivada da CPC, pertencente à União Européia, sendo gerida pelo Statistical Office of the European Communities. A TAB. 8 mostra a estrutura hierárquica da CPA.

TABELA 8

Estrutura da Classificação CPA (Statistical Classification of Products by Activity in the European Economic Community)

\begin{tabular}{lcc}
\hline \multicolumn{1}{c}{ Nível } & Codificação & oo de itens \\
\hline \hline Seções & 1 caractere alfabético (A-Q) & 17 \\
Subseções & 2 caracteres alfabéticos & 31 \\
Divisões & 2 dígitos & 62 \\
Grupos & 3 dígitos & 223 \\
Classes & 4 dígitos & 502 \\
Categorias & 5 dígitos & 1.146 \\
Subcategorias & 6 dígitos & 2.608
\end{tabular}

Fonte: União Européia (2007f). 
A seguir, exemplifica-se o sistema de classificação CPA (QUADRO 8). QUADRO 8

Exemplificação do processo de codificação do Sistema de Classificação CPA (Statistical

Classification of Products by Activity in the European Economic Community) aplicado à Indústria Química

D - Produtos da Indústria de transformação (SEÇÃO)

DG - Produtos da indústria química e fibras artificiais e sintéticas (SUBSEÇÃO)

24 - Produtos da indústria química e fibras artificiais e sintéticas (DIVISÃO)

24.1 - Produtos químicos básicos (GRUPO)

24.11 - Gases industriais (Classes)

24.11.1 - Gases industriais (Categorias)

24.11.11 - Hidrogênio, gases raros, nitrogênio e oxigênio

(Subcategorias)

Fonte: Elaborado a partir de União Européia (2007g).

Embora derivada da CPC, a estrutura utilizada na Statistical Classification of Products by Activity in the European Economic Community (CPA) difere da sua classificação-referência, por utilizar caracteres alfabéticos e um dígito a mais (totalizando seis) em sua codificação, pois a CPC utiliza um sistema exclusivo de cinco dígitos. Os produtos químicos encontram-se distribuídos ao longo da Divisão CPA 24, pertencente à Subseção CPA DG (Produtos da indústria química e fibras artificiais e sintéticas) da Seção CPA D (Produtos da Indústria de transformação).

No Grupo CPA 24.1 (Produtos químicos básicos), diferente das demais classificações de produtos já apresentadas, não somente os compostos químicos orgânicos básicos encontram-se listados em conjunto com os inorgânicos básicos, mas também são descritos no mesmo grupo as tinturas e pigmentos (Classe 24.12), fertilizantes (Classe 24.15), plásticos em suas formas primárias (Classe 24.16) e borrachas sintéticas em suas formas primárias (Classe 24.17), ressalvando os compostos químicos localizados nas outras classes.

Os pesticidas e outros agroquímicos, além dos produtos antibrotamento, reguladores do crescimento de plantas e desinfetantes agrícolas, encontram-se no Grupo CPA 24.2. O Grupo CPA 24.3 trata das tintas e vernizes em conjunto com os revestimentos, tintas de impressão e mastiques. Outros compostos químicos corantes são encontrados na Categoria 24.12.2, e as tintas de impressão e tintas para escrever e desenhar estão na Categoria 24.66.2.

Os produtos químico-farmacêuticos e de uso medicinal são abordados no Grupo CPA 24.4. O Grupo CPA 24.5 aborda conjuntamente os sabões, detergentes e preparações de limpeza, cosméticos, perfumes e preparações de toalete. Na CPA 24.51.41 são abordadas as preparações para desodorização ou odorização de ambientes, e na Classe CPA 24.52 os perfumes e preparações de toalete. 
O Grupo CPA 24.7 refere-se às fibras artificiais e sintéticas. A Categoria CPA 24.14.7 e o Grupo 24.6 tratam dos demais produtos químicos não listados nos campos anteriormente citados.

Outras Subseções da CPA privilegiam produtos acabados elaborados a partir dos compostos químicos descritos nos grupos e classes dos parágrafos anteriores (Subseções DH a DJ). Os produtos petroquímicos derivados do refino do petróleo encontram-se distribuídos no Grupo CPA 23.2 da Subseção DF.

\section{Conclusões}

Percebe-se a variedade de classificações às quais os produtos químicos são subordinados. De modo geral, as classificações de atividades econômicas e produtos não são excludentes entre si, mas podem sobrepor-se umas às outras com relativa facilidade e mínima intervenção. Devido aos diferentes níveis hierárquicos das classificações integrantes da "Família Internacional", as informações colhidas são processadas em um grau de detalhamento maior ou menor, refletindo na adoção de níveis de codificação hierárquica mais ou menos extensos, que podem conter uma quantidade maior ou menor de dígitos para cada código adotado. As nomenclaturas derivadas, de uma forma geral, são as que apresentam a maior quantidade de itemizações, por terem um maior número de níveis e de dígitos empregados.

Todas essas diferenças fazem com que seja extremamente complexa e extenuante a própria tentativa do profissional da área da química em criar divisões para as diferentes classes de produtos químicos existentes, quanto à procura de um denominador comum que permita comparar entre si as classificações existentes na atualidade.

\section{Referências}

BORSCHIVER, S.; WONGTSCHOWSKI, P.; ANTUNES, A classificação industrial e sua importância na análise setorial. Ciência da Informação, Brasília, v.33, n.1, p.9-21, jan./abr. 2004.

BORSCHIVER, S. Estudo do Impacto da Indústria Química na Economia através do Sistema de Contas Nacionais. Rio de Janeiro, 2002. 335 p. Tese (Doutorado em Engenharia Química) - Escola de Química, Universidade Federal do Rio de Janeiro - EQ/UFRJ, Rio de Janeiro.

COMISSÃO NACIONAL DE CLASSIFICAÇÃO - CONCLA (Brasil). Comissão Nacional de Classificação. Subcomissão Técnica da CNAE-Fiscal: Estrutura. Disponível em: <http://200.189.113.39/sitecnae.nsf>. Acesso em: 19 mar. 2007.

MERCOSUL. MERCOSUL/GMC/RES. $N^{\circ}$ 70/06: tarifa externa comum incorporação à NCM da IV emenda ao sistema harmonizado de designação e codificação de mercadorias. Disponível em: <http://www.mercosur.int/msweb/SM/Actas\%20TEMPORARIAS/GMC/LXV I\%20GMC\%20ACTA\%2005- 
06\%20FINAL/NORMAS\%20y\%20Projetos/NORMAS\%20APROBADAS\%20L XVI\%20GMC/RESOLU\%C3\%87\%C3\%95ES/GMC_Res_\%2070-

06\%20PT\%20-\%20IV\%20Enmienda.pdf>. Acesso em: 19 mar. 2007.

NAÇÕES UNIDAS (New York). United Nation Economic Statistics and Classifications Section: International Standard Industrial Classification of All Economic Activities. Revision 4. Disponível em: <http://unstats.un.org/unsd/cr/family2.asp?Cl=27> . Acesso em: 14 mar. 2007a.

code. Detailed structure and explanatory notes of ISIC Rev.4 (draft) $<$ http://unstats.un.org/unsd/cr/registry/regcst.asp? $\mathrm{Cl}=27 \& \mathrm{Lg}=1>$. Acesso em: 14 mar. 2007b.

. The North American Industry Classification System United States 2002, (NAICS-US 2002). Disponível em: $<$ http://unstats.un.org/unsd/cr/family2.asp?Cl=230> . Acesso em: 14 mar. 2007c.(c)

- Central Product Classification Version 1.1, (CPC Ver. 1.1). Disponível em: <http://unstats.un.org/unsd/cr/family2.asp? $\mathrm{Cl}=16>$. Acesso em: 19 mar. 2007d.

Disponível

Detailed structure and explanatory notes of CPC Ver. 1.1 code 3. $<$ http://unstats.un.org/unsd/cr/registry/regcst.asp?Cl=16>. Acesso em: 14 mar. 2007e.

. Statistical Papers Series M No. 34/Rev. 4 Standard International

Trade Classification Revision 4. Disponível em:
$<$ http://unstats.un.org/unsd/trade/SITC\%20Rev\%204\%20FINAL.pdf>.

Acesso em: 19 mar. $2007 f$.

UNIÃO EUROPÉIA. General Description of Classification item 70, NACE Rev 2.

$<$ http://ec.europa.eu/eurostat/ramon/nomenclatures/index.cfm?TargetUrl $=$ LST NOM\&StrLanguageCode $=E N \& I n t F a m i l y C o d e=\& T x t S e a r c h=\& I n t C u r r$ entPage $=2>$. Acesso em: 14 mar. 2007a.

. Statistical Classification of Economic Activities in the European Community, Rev. 2 (2007) (NACE Rev. 2). Disponível em: $<$ http://ec.europa.eu/eurostat/ramon/nomenclatures/index.cfm?TargetUrl =ACT OTH CLS DLD\&StrNom=NACE REV2\&StrFormat=HTML\&StrLangua geCode $=E N \& I n t K e y=18496334>$. Acesso em: 14 mar. 2007b.

. General Description of Classification item 39, HS 2002. Disponível em

$<$ http://ec.europa.eu/eurostat/ramon/nomenclatures/index.cfm?TargetUrl $=$ LST NOM\&StrLanguageCode $=E N \& I n t F a m i l y C o d e=\& T x t S e a r c h=\& I n t C u r r$ entPage $=1>$. Acesso em: 19 mar. 2007c. 
- Harmonized Commodity Description and Coding System, 2002 (HS 2002; Harmonized System 2002). Disponível em: $<$ http://ec.europa.eu/eurostat/ramon/nomenclatures/index.cfm?TargetUrl $=$ ACT OTH CLS DLD\&StrNom=HS 2002\&StrFormat=HTML\&StrLanquage Code $=$ EN\&IntKey=5525517 > . Acesso em: 19 mar. 2007d.

Disponível

General Description of Classification item 66, SITC Rev 4.

$<$ http://ec.europa.eu/eurostat/ramon/nomenclatures/index.cfm?TargetUrl =LST NOM\&StrLanguageCode=EN\&IntFamilyCode=\&TxtSearch=\&IntCurr entPage $=2>$. Acesso em: 19 mar. $2007 \mathrm{e}$.

Disponível

General Description of Classification item 72, CPA 2002.

$<$ http://ec.europa.eu/eurostat/ramon/nomenclatures/index.cfm?TargetUrl $=$ LST NOM\&StrLanguageCode $=E N \& I n t F a m i l y C o d e=\& T x t S e a r c h=\& I n t C u r r$ entPage $=2$ > . Acesso em: 19 mar. $2007 \mathrm{f}$.

. Statistical Classification of Products by Activity in the European Economic Community, 2002 version (CPA 2002). Disponível em: $<$ http://ec.europa.eu/eurostat/ramon/nomenclatures/index.cfm?TargetUrl =ACT OTH CLS DLD\&StrNom=CPA\&StrFormat $=$ HTML\&StrLanguageCode $=$ EN\&IntKey=839094 >. Acesso em: 19 mar. 2007g.

U.S. CENSUS BUREAU (Washington). 2002 NAICS Codes and Titles. Disponível em: <http://www.census.gov/epcd/naics02/naicod02.htm>. Acesso em: 14 mar. 2007. 